\title{
Comparison Between Two Methods of Bioelectrical Impedance Analyses for Measuring Abdominal Visceral Fat Tissue
}

\author{
Hidetaka Hamasaki ${ }^{a}$,, Yasuteru Hamasaki ${ }^{a}$
}

\begin{abstract}
Background: The assessment of visceral adiposity is essential for the management of obesity. Bioelectrical impedance analysis (BIA) has been established as a practical method for the assessment of visceral adiposity.
\end{abstract}

Methods: We compared two BIA methods (seca, mBCA 515; Omron, HDS-2000) of assessing visceral adiposity.

Results: Visceral adipose tissue volume measured using seca mBCA 515 was highly correlated with visceral fat area measured using HDS2000; however, they were differently correlated with waist circumference and body mass index, respectively.

Conclusions: The seca mBCA 515 can distinguish fat mass from lean mass more accurately compared with conventional BIA methods.

Keywords: Obesity; Visceral adiposity; Bioelectrical impedance analysis; Body mass index; Waist circumference

\section{Introduction}

Visceral adiposity is associated with an increased risk of cardiovascular disease [1]; therefore, the simple, practical, and accurate assessment of visceral adiposity is essential for the prevention and management of obesity. Bioelectrical impedance analysis (BIA) has been established as a practical method for the assessment of visceral adiposity as well as body composition; however, its validity has not been fully verified $[2,3]$. Recently, a novel medical BIA device (seca, medical Body Composition Analyzer 515; seca mBCA 515) has been developed [4]. Body composition predicted using this BIA device was highly correlated with that measured by mag-

Manuscript submitted June 22, 2018, accepted July 2, 2018

aHamasaki Clinic, Kagoshima, Japan

${ }^{b}$ Corresponding Author: Hidetaka Hamasaki, Hamasaki Clinic, 2-21-4 Nishida, Kagoshima 890-0046, Japan. Email: hhamasaki78@gmail.com

doi: https://doi.org/10.14740/jem518w netic resonance imaging (MRI). The authors concluded that BIA equations based on MRI measurement are more accurate for predicting skeletal muscle mass than those based on dual energy X-ray absorptiometry. In addition, visceral adipose tissue volumes (VAT) predicted using this BIA device were also highly correlated with VAT $\left(\mathrm{r}^{2}=0.8\right)$ evaluated by MRI [4]. On the other hand, another BIA device (Omron, HDS2000) has also been used for measuring the visceral fat area (VFA), and the result shows a strong correlation $(\mathrm{r}=0.89)$ with VFA measured by computed tomography (CT) [5]. Using seca mBCA 515, VAT can be measured in a standing position within $17 \mathrm{~s}$, whereas it takes several minutes for measuring VFA by HDS-2000 in a supine position. If clinicians can accurately assess visceral adiposity using seca mBCA 515, it will be useful for the management of obesity. The aim of this study was to compare these two BIA methods of assessing visceral adiposity.

\section{Methods}

\section{Subjects}

We conducted a cross-sectional study in subjects who visited our clinic. First, height and weight were measured; waist circumference (WC) was also measured at the umbilical level in a standing position at the end of exhalation. Second, body composition was measured using seca mBCA 515. Third, VFA was measured using HDS-2000 in a supine position. All the measurements were performed in a fasting state on the same morning. The study protocol was approved by the Medical Ethics Committee of the Japan Medical Association, Center for Clinical Trials (Reference No.29-6), and the study was performed in accordance with the Declaration of Helsinki.

\section{Statistical analysis}

The statistical analyses were performed using SPSS version 24 (IBM Co., Ltd, Chicago, USA). All continuous values were expressed as the mean \pm standard deviation. Correlations among VAT, VFA, weight, body mass index (BMI), and WC were calculated using the Pearson's correlation coefficient. $\mathrm{P}$ value $<0.05$ was considered to be statistically significant. 


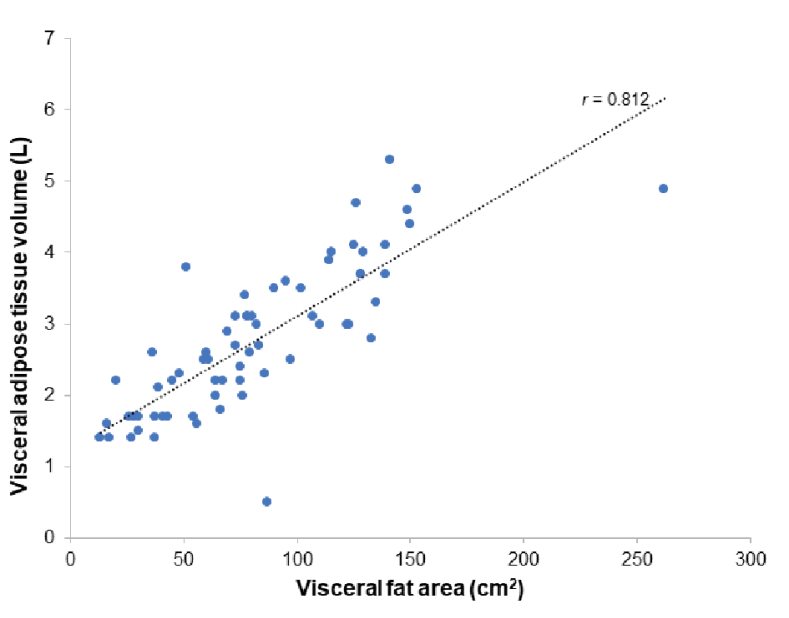

Figure 1. Correlation between visceral fat tissue volume measured by seca mBCA 515 and visceral fat area measured by HDS-2000.

\section{Results}

A total of 66 subjects ( 39 males and 27 females) were enrolled in this study. Overall, 48 subjects were diagnosed as having type 2 diabetes and 18 subjects were healthy individuals. The mean age and BMI were $59.2 \pm 14.9$ and $24.5 \pm 4.4 \mathrm{~kg} / \mathrm{m}^{2}$, respectively. The correlation between VAT and VFA was high (Pearson's correlation coefficient; $r=0.812, \mathrm{P}<0.001$ ) (Fig. 1). VAT was significantly correlated with VFA in subjects with type 2 diabetes $(\mathrm{r}=0.755, \mathrm{P}<0.001)$ as well as in healthy subjects $(\mathrm{r}=0.829, \mathrm{P}<0.001)$. Interestingly, VAT was more strongly correlated to WC than to VFA. On the other hand, VFA was more strongly correlated to BMI than to VAT (Table 1).

\section{Discussion}

This study demonstrates that a novel BIA device can measure visceral adiposity that shows a high correlation with weight, BMI, WC, and VFA measured by another BIA device. To our knowledge, this is the first study to compare the two methods of BIA for evaluating visceral adiposity. The inability of BMI to distinguish between fat and lean mass has been recognized, and the obesity paradox has also been reported in subjects who suffer from chronic conditions such as diabetes, cancer and renal disease [6]. In contrast, seca mBCA 515 can accurately measure body composition in patients with a chronic disease such as cancer or renal failure $[7,8]$. The finding of this study that VAT had a higher correlation with WC than with BMI suggests that seca mBCA 515 can distinguish fat mass from lean mass more accurately compared with conventional BIA methods. Total VAT measurement has been considered as the golden standard, and a single-slice VFA assessment may not accurately evaluate visceral adiposity in obese individuals [9]. Because it is rapid, noninvasive, and accurate, seca mBCA 515 may be useful for evaluating visceral adiposity in subjects with chronic disease. However, this study was a single-center, small number, cross-sectional study. Further study is warranted to
Table 1. Correlations of Visceral Adiposity With Weight, BMI, and WC

\begin{tabular}{lll}
\hline & r & P \\
\hline VFA (measured by HDS-2000) & & \\
Weight & 0.742 & $<0.001$ \\
BMI & 0.841 & $<0.001$ \\
WC & 0.839 & $<0.001$ \\
VAT (measured by seca BCA 515) & & \\
Weight & 0.726 & $<0.001$ \\
BMI & 0.779 & $<0.001$ \\
WC & 0.909 & $<0.001$ \\
\hline
\end{tabular}

BMI: body mass index; WC: waist circumference; VFA: visceral fat area; VAT: visceral adipose tissue volume.

investigate the usefulness of making the device available in various clinical settings.

\section{Acknowledgments}

The authors appreciate the support of Yuka Miyaji for data collection.

\section{References}

1. Mathieu P, Boulanger MC, Despres JP. Ectopic visceral fat: a clinical and molecular perspective on the cardiometabolic risk. Rev Endocr Metab Disord. 2014;15(4):289298.

2. Thomas EL, Collins AL, McCarthy J, Fitzpatrick J, Durighel G, Goldstone AP, Bell JD. Estimation of abdominal fat compartments by bioelectrical impedance: the validity of the ViScan measurement system in comparison with MRI. Eur J Clin Nutr. 2010;64(5):525-533.

3. Ward LC. Segmental bioelectrical impedance analysis: an update. Curr Opin Clin Nutr Metab Care. 2012;15(5):424429.

4. Bosy-Westphal A, Jensen B, Braun W, Pourhassan M, Gallagher D, Muller MJ. Quantification of whole-body and segmental skeletal muscle mass using phase-sensitive 8-electrode medical bioelectrical impedance devices. Eur J Clin Nutr. 2017;71(9):1061-1067.

5. Park KS, Lee DH, Lee J, Kim YJ, Jung KY, Kim KM, Kwak SH, et al. Comparison between two methods of bioelectrical impedance analyses for accuracy in measuring abdominal visceral fat area. J Diabetes Complications. 2016;30(2):343-349.

6. Prado CM, Gonzalez MC, Heymsfield SB. Body composition phenotypes and obesity paradox. Curr Opin Clin Nutr Metab Care. 2015;18(6):535-551.

7. Raeder H, Kvaerner AS, Henriksen C, Florholmen G, Henriksen HB, Bohn SK, Paur I, et al. Validity of bioelectrical impedance analysis in estimation of fat-free mass in colorectal cancer patients. Clin Nutr. 2018;37(1):292- 
300 .

8. Slowinski T, Lieker I, Otto B, Khadzhynov D, Schuetz M, Neumayer H-H. Pilot study on the accuracy of bioimpedance analysis in detection of changes of body fluid composition in patients during a hemodialysis session. The 4th annual meeting of the German Society for Nephrol- ogy, October 2012.

9. Maislin G, Ahmed MM, Gooneratne N, Thorne-Fitzgerald M, Kim C, Teff K, Arnardottir ES, et al. Single slice vs. volumetric MR assessment of visceral adipose tissue: reliability and validity among the overweight and obese. Obesity (Silver Spring). 2012;20(10):2124-2132. 